Number of words in manuscript (excluding abstract): $\quad 3,808$

Number of tables: $\quad 5$

Number of figures: 2

\title{
Association between a longer duration of illness, age and lower frontal lobe grey matter volume in schizophrenia
}

Preethi Premkumar $^{\mathrm{a}, *}$, Dominic Fannon ${ }^{\mathrm{b}}$, Elizabeth Kuipers ${ }^{\mathrm{a}, \mathrm{d}}$, Michael A Cooke ${ }^{\mathrm{a}}$, Andrew Simmons ${ }^{c, d}$, Veena Kumari ${ }^{\text {a }}$

${ }^{a}$ Department of Psychology; ${ }^{b}$ Division of Psychological Medicine and Psychiatry, \& ${ }^{c}$ Centre for Neuroimaging Sciences, Institute of Psychiatry, King's College London, London, United

Kingdom; d NIHR Biomedical Research Centre for Mental Health, South London and Maudsley Foundation NHS Trust, London, United Kingdom

Running title: Duration of illness and grey matter in schizophrenia

Correspondence to:

Preethi Premkumar

Department of Psychology

Institute of Psychiatry

De Crespigny Park

London SE5 8AF

Tel: +442078485102

Fax: +44 2078480860

E.mail: Preethi.premkumar@iop.kcl.ac.uk 


\section{Abstract}

The frontal lobe has an extended maturation period and may be vulnerable to the long-term effects of schizophrenia. We tested this hypothesis by studying the relationship between duration of illness (Dol) and grey matter (GM) volume across the whole brain. Sixty-four patients with schizophrenia and 25 healthy controls underwent structural MRI scanning and neuropsychological assessment. We performed regression analyses in patients to examine the relationship between Dol and GM and cerebro-spinal fluid (CSF) volumes across the whole brain, and correlations in controls between age and GM or CSF volume of the regions where GM or CSF volumes were associated with Dol in patients. Correlations were also performed between GM volume in the regions associated with Dol and neuropsychological performance. A longer Dol was associated with lower GM volume in the left dorsomedial prefrontal cortex (PFC), right middle frontal cortex, left fusiform gyrus (FG) and left cerebellum (lobule III). Additionally, age was inversely associated with GM volume in the left dorsomedial PFC in patients, and in the left FG and CSF excess near the left cerebellum in healthy controls. Greater GM volume in the left dorsomedial PFC was associated with better working memory, attention and psychomotor speed in patients. Our findings suggest that the right middle frontal cortex is particularly vulnerable to the long-term effect of schizophrenia illness whereas the dorsomedial PFC, FG and cerebellum are affected by both a long Dol and aging. The effect of illness chronicity on GM volume in the left dorsomedial PFC may be extended to brain structureneuropsychological function relationships. 


\section{Introduction}

The prefrontal cortex (PFC) plays a key role in most neuropsychological functions, being associated with working memory, problem-solving, decision-making, response inhibition, reward and attention [1]. From an evolutionary perspective, it is regarded as a higher-order brain structure, forming a part of the neo-cortex [2].

In schizophrenia, there is reduced PFC volume compared to healthy populations [3]. This characteristic, in addition to an impairment in most frontal lobe-based neuropsychological functions, such as verbal fluency, set-shifting, $n$-back tasks, vigilance and response inhibition [4], would suggest that the PFC is important to the psychopathology of schizophrenia [5]. The frontal lobe has a maturation period extending into the fifth decade [6]. While some brain structural changes are reported early in the course of schizophrenia [7-11], the longer time to maturation of the frontal lobe would render it more vulnerable to the emergence and course of schizophrenia later in the course of schizophrenia illness. Notably, a post-mortem study of schizophrenia patients ( $n=14$, mean age $48.5 \pm 16$ years) and individuals without a history of mental illness ( $n=19$, mean age $55 \pm 15.3$ years) found smaller grey matter (GM) volume in the frontal but not other cerebral lobes in schizophrenia [12].

Longitudinal studies have reported frontal lobe volume reduction in patients with schizophrenia [13-15]. Studies have also examined the cross-sectional relationship between the duration of illness and frontal lobe volume [16-18]. Molina and colleagues [18] observed a loss of GM in the PFC in chronic ( $n=29)$, but not first-episode $(n=22)$, schizophrenia patients and an association between cerebro-spinal fluid (CSF) excess in the PFC and duration of illness in male, but not female, patients [19]. Smaller PFC GM volume has been reported to be associated non-linearly with a longer duration of illness in chronic $(n=49)$ and first-episode $(n=$ 34) patients [16]. Less GM in the superior part of the dorsolateral PFC (DLPFC) has been associated with a longer duration of illness in chronic $(n=21)$, but not first-episode $(n=22)$, 
patients [20]. Longer duration of illness has also been associated with reduced GM in left temporal lobe and right posterior region, but not frontal lobe in neuroleptic-naïve first-episode patients $(n=14)$ [21]. More recently, another study [17] observed an inverse association between duration of illness and prefrontal cortical volume in stable outpatients $(n=28)$. A lower $\mathrm{N}$-acetyl aspartate/Creatine ratio in the DLPFC is also associated with a longer duration of illness among schizophrenia patients $(n=35)$ [22]. A loss of PFC GM and NAA thus seems to be associated with a longer duration of illness, perhaps more reliably in the chronic than early course, in schizophrenia.

Studying GM volume using voxel-based morphometry (VBM) can help detect the localized effects of duration of illness in schizophrenia patients. GM volume decrease in frontal and temporal regions over a five-year period has been observed, with a progressive GM loss in the left frontal lobe as the number of psychotic episodes increased [23]. One study [24] of 20 schizophrenia patients (mean age $=37$ years, median duration of illness $=15$ years [2-41 years]) did not observe any association between duration of illness and GM volume across the brain. Another study [25] $(n=39)$ reported (mean age $=36$ years, median duration of illness $=$ 11 years [2-31 years]) an inverse association between duration of illness and GM volume in the medial temporal lobe, cerebellum and the anterior cingulate gyrus, but not in the frontal lobe. GM volume in the frontal and temporal lobes, as determined by $\mathrm{ROI}[26,27]$ and more recently by VBM $[28,29]$ approaches, has also been found to change with age in healthy individuals.

In this study, we examined the inverse association between duration of illness and GM volume as determined by VBM in a larger sample of patients with schizophrenia $(n=64)$ than previous studies that examined this association. We hypothesized that a longer duration of illness would be related to lower GM volume in the PFC. We, however, examined the relationship between the duration of illness and GM and CSF volumes across the whole brain to determine the specificity of a duration of illness-PFC volume association (if found). In order to 
understand the cognitive and clinical factors associated with duration of illness, we also examined the association of duration of illness to executive function, memory, attention and motor dexterity and positive and negative symptoms and generalized psychopathology. Based on previous findings [30-32], we hypothesized that a longer duration of illness would be associated most strongly with poorer PFC-based cognitive function.

\section{Methods and Materials}

\subsection{Participants and design}

Participants included 64 outpatients with a DSM-IV diagnosis of schizophrenia or schizoaffective disorder who were recruited as part of a longitudinal study on the effect of cognitive behavioural therapy (CBT) on the brain and 25 healthy controls. The present investigation utilized patients' data collected at baseline (prior to CBT). Patients were recruited from the South London and Maudsley NHS Trust and were on stable doses of antipsychotic medication for at least three months prior to taking part. Healthy participants were recruited from the local community and screened for personal history of mental illness using the SCID non-patient version [33]. No participant had a history of neurological or systemic illness or head injury. The study procedures and the use of data for the purpose of the current investigation were approved by the ethics committee of the joint research ethics committee of the South London and Maudsley NHS Foundation Trust and the Institute of Psychiatry, London. All participants provided written informed consent to their participation and were compensated for their time and travel.

All participants underwent magnetic resonance imaging and were tested on a battery of neuropsychological tests. 


\subsection{Clinical assessments}

Clinical diagnostic interviews using the Structured Clinical Interview for DSM-IV (SCID) [34], and Positive and Negative Syndrome Scale (PANSS) assessments [35] were performed by an experienced consultant psychiatrist (DF). Duration of illness was defined as the difference between the age at onset of psychotic symptoms (as reported by the patient and where possible confirmed with other sources) and age at the time of scanning.

\subsection{Neuropsychological measures}

Participants were assessed on general intelligence and measures of executive function, memory, attention and motor dexterity (see Table 1).

Insert Table 1 about here

\subsection{MRI acquisition}

Structural MRI brain scans were acquired using a 1.5 Tesla GE NV/I Signa system (General Electric, Milwaukee WI, USA) at the Maudsley Hospital, London. Initially, a series of sagittal fast gradient echo scout images were acquired. A 3-D inversion recovery prepared fast spoiled GRASS sequence was applied to the whole brain to obtain T1-weighted images in the axial plane with $1.5 \mathrm{~mm}$ contiguous sections $(\mathrm{TR}=18 \mathrm{~ms}, \mathrm{TI}=450 \mathrm{~ms}, \mathrm{TE}=5.1 \mathrm{~ms}$, flip angle $=$ 20 degrees with one data average and a $256 \times 256 \times 128$ voxel matrix). 


\subsubsection{MRI pre-processing}

Structural images were converted into ANALYZE format (ANALYZE software, BRU, Mayo Foundation, Rochester, MN) and pre-processed using Statistical Parametric Mapping version 2 (SPM2, Wellcome Department of Imaging Neuroscience, London; http://www.fil.ion.ucl.ac.uk/spm), running in MATLAB 6.1 (MathWorks, Natick, MA). All images were manually realigned (three translations: right, forward and up; and three rotations: pitch, roll and yaw) to the anterior commissure-posterior commissure (AC-PC) line and the interhemispheric fissure.

\subsubsection{Customised template creation}

Customised T1-weighted templates of the whole brain, GM, WM and cerebro-spinal fluid (CSF) were created for patients and controls separately. For the creation of the customised whole brain (T1-weighted) template, the images were spatially normalized to the standard SPM2 T1-weighted template using a 12-parameter affine transformation. These normalised images were then smoothed with an 8mm full width at half maximum (FWHM) isotropic Gaussian kernel, and averaged to create a customised T1-weighted template. The normalised T1-weighted images were then segmented into their GM, WM and CSF components using the GM, WM and CSF probability maps inherent to SPM2. The resultant tissue segments were automatically cleaned to remove non-brain tissue and smoothed with an 8mm FWHM isotropic Gaussian kernel, normalized using affine transformation with sinc interpolation algorithm and averaged to derive GM, WM and CSF probability maps. To reduce the partial volume problem and ensure optimal tissue segmentation, all images for the templates were written out with $1 \times 1 \times 1 \mathrm{~mm}$ voxel size. 


\subsubsection{Deriving and applying optimized normalization parameters}

The structural scans were processed using the customized whole brain and tissue probability templates. The first step entailed a segmentation of the original images in native space, registering to the customized tissue probability map and correcting for image inhomogeneity, followed by an automatic brain extraction and cleaning procedure to remove non-brain tissue. The second step involved spatial normalization of the original images to the customized whole brain template using 12-parameter linear and $7 \times 8 \times 7$ discrete cosine transform basis function non-linear transformation [36], with parameters determined from the images derived from the first step, and resliced to $1 \times 1 \times 1 \mathrm{~mm}$ voxel size to yield more accurate subsequent tissue segmentation. The spatially normalized images were then segmented into the three tissue compartments using the customized GM, WM and CSF templates. Brain extraction and cleaning procedures were re-applied to the segmented normalized GM images to further remove extraneous brain tissue. Since the volume of some brain regions may shrink or expand as a result of non-linear spatial normalization, the cleaned GM images were modulated, i.e., the voxel values of each segment were multiplied by the Jacobian determinants of the deformation matrix derived during the spatial normalization step to 'restore' the original volume of each GM segment. Finally, the GM and WM segments were smoothed using a 12-mm FWHM isotropic Gaussian kernel to make the data conform to the Gaussian field model, underlying the structural inferences as implemented in SPM2 to render the data more normally distributed (by the central limit theorem) and to reduce the effects of individual variation in sulcal/gyral anatomy [37].

\subsection{Statistical analysis}

\subsubsection{Group comparison on demographic characteristics}


Group differences in demographic characteristics were examined with analysis of variance (ANOVA) and Chi-square tests.

\subsubsection{Group comparison on neuropsychological performance}

Differences between patients and controls in neuropsychological performance with age and years in education as covariates (because these variables differentiated the study groups; see Table 1) were examined by means of one-way analyses of covariance (ANCOVAs).

\subsubsection{Neuropsychological and clinical correlates of duration of illness}

The associations between duration of illness and (a) neuropsychological ability and (b) total PANSS and PANSS total positive, negative and general psychopathology subscale scores were determined with Pearson's correlations. Given that we expected only small-to-modest associations between duration of illness and poor cognitive function and the exploratory nature of the analysis concerning the relationship between duration of illness and symptoms, the significance level was set at $p=0.05$ and not corrected for multiple correlations.

\subsubsection{MRI analysis}

\subsubsection{Effect of diagnosis on GM and CSF volume}

Reduction in GM volume and increase in CSF were explored across the whole brain in patients compared to controls by means of ANCOVAs at each voxel using SPM2 with diagnosis as a between-group factor, and age as a confounding covariate. The group difference map in GM volume across the whole brain was examined at an uncorrected height threshold $p$ level < 0.001 ; the differences which survived family-wise error correction at $p$ level $<0.05$ were considered significant. 


\subsubsection{Association of GM or CSF volume with duration of illness and age in patients and with age in healthy controls}

The association between GM or CSF volume and duration of illness in patients was examined by means of multiple regressions between GM or CSF maps and duration of illness at each voxel.

In order to disentangle the effects of age and duration of illness, we performed multiple regression analyses in patients with GM or CSF volume at each voxel of the regions where GM or CSF volumes were associated with duration of illness as the dependent variable and age and duration of illness as the predictor variables in order to determine which was the stronger predictor and the variance predicted by the two variables.

We examined separately the association between age and GM or CSF volume in controls at the maxima voxels of all the regions where GM or CSF volumes were associated with duration of illness in patients by means of correlations between the values representing the percentage of total grey/white matter volume under a smoothing kernel relative to the total grey/white matter volume extracted for each participant and age at each voxel.

We further examined the GM volume association with duration of illness in patients with a schizophrenia diagnosis only $(n=56)$.

\subsubsection{GM volume, duration of illness and neuropsychological performance in} patients

We extracted the values representing the percentage of total grey/white matter volume under a smoothing kernel relative to the total grey/white matter volume for each participant in the patient group at the maxima voxels of all the regions where GM volumes were associated 
with duration of illness in patients. We performed Pearson correlations between these values and performance scores on the neuropsychological measures.

\section{Results}

Patients had a fewer number of years in education (see Table 2). Fifty-three patients were receiving atypical (clozapine $=10$, risperidone $=10$, olanzapine $=22$, quetiapine $=2$, amisulperide $=2$, aripiprazole=2), eleven were receiving typical (chlorpromazine $=1$, flupenthixol=3, sulperide $=3$, haloperidol $=2$, fluphenazine $=1$, thioridazine $=1$ ) and three were receiving both atypical and typical antipsychotics.

Insert Table 2 about here

\subsection{Effect of diagnosis on neuropsychological performance}

Patients had lower IQ and performed poorly relative to healthy controls on most neuropsychological measures (see Table 3).

Insert Table 3 about here

\subsection{Neuropsychological and clinical correlates of duration of illness}

Duration of illness did not correlate with any neuropsychological measure; the strongest but still non-significant correlation was with general intelligence $(r=-0.17, p=0.19)$. 
Duration of illness was not significantly associated with current positive symptoms ( $r=-$ $0.02, p=0.87)$, negative symptoms $(r=0.02, p=0.91)$, general psychopathology $(r=-0.06, p=0.64)$ or total PANSS scores $(r=-0.06, p=0.64)$.

\subsection{Effect of diagnosis on GM and CSF volume across the whole brain}

Patients had lower GM volume in the left and right dorsolateral PFC, left and right middle temporal gyrus and left anterior cingulate gyrus (see Table 4 and Figure 1). The GM reduction was greatest in the left dorsolateral PFC. A CSF excess in patients was present near the superior frontal gyrus bilaterally, right dorsolateral PFC, left superior temporal gyri and right precentral gyrus.

Insert Table 4 and Figure 1 about here

\subsection{GM correlates of duration of illness in patients and age in healthy controls}

A longer duration of illness was associated with lower GM volume in the left dorsomedial PFC, right middle frontal cortex, left fusiform gyrus (FG) and left cerebellum (see Table 5 and Figure 2) and with a CSF excess near the left cerebellum. Among patients with a schizophrenia diagnosis only, a longer duration of illness was associated with lower GM volume in the left dorsomedial PFC and right middle frontal cortex and a CSF excess near the left inferior frontal gyrus. 
Duration of illness predicted $19.6 \%$ of the variance in GM volume of the left dorsomedial PFC $(r=-0.44, p<0.001)$, while age predicted a further $6 \%$ of the variance (partial $r=-0.32$, $p=0.04$, overall $\mathrm{F}=10.3, \mathrm{df}=63,2, p<0.001)$.

Duration of illness predicted $16.7 \%$ of the variance in GM volume of the right middle frontal cortex $(r=-0.41, p=0.001)$, while age predicted a further $0.01 \%$ (negligible) of the variance (partial $r=0.04, p=0.78$, overall $F=6.15, \mathrm{df}=63,2, p=0.004$ ).

Duration of illness predicted $16.8 \%$ of the variance in GM volume of the left fusiform gyrus $(r=-0.41, p=0.001)$, while age predicted a further $0.03 \%$ of the variance (partial $r=-0.08$, $p=0.61$, overall $F=6.29, \mathrm{df}=63,2, p=0.003)$.

Duration of illness predicted $16 \%$ of the variance in GM volume of the left cerebellum (lobule III) $(r=-0.4, p=0.001)$, while age predicted a further $2 \%$ of the variance (partial $r=0.2$, $p=0.2$, overall $F=6.77, d f=63,2, p=0.002)$.

In healthy controls, age was associated with GM volume in the left FG $(r=-0.39, p=0.05)$ and with CSF volume near the cerebellum $(r=0.48, p=0.02)$ and at a trend level with GM volume in the left dorsomedial PFC (partial $r=-0.37, p=0.07$ ).

Insert Table 5 and Figure 2 about here

\subsection{GM volume, duration of illness and neuropsychological performance in patients}

Greater GM volume in the dorsomedial PFC was associated with better performance on the letter-number test $(r=0.3, p=0.02)$, Stroop Interference $(r=0.38, p=0.002)$ and shorter time to completion on the Groove pegboard dominant task $(r=-0.25, p=0.04)$. 


\section{Discussion}

We aimed to determine whether a longer duration of illness is related to GM loss and CSF excess in the PFC in patients with schizophrenia. Our study found that longer duration of illness was associated with lower GM volume in the left dorsomedial prefrontal and right middle frontal cortices, left FG and left cerebellum (lobule III) in patients with schizophrenia or schizoaffective disorder. GM volume in the left dorsomedial PFC was influenced by age in addition to the duration of illness. In schizophrenia patients only, duration of illness was associated with lower GM volume in the left dorsomedial prefrontal and right middle frontal cortices and with CSF excess near the left inferior frontal gyrus. In controls, age was inversely associated with GM volume in the left FG and CSF excess near the cerebellum, and at a trend level with GM volume in the left dorsomedial PFC. Compared to controls, patients had lower GM volume bilaterally in the DLPFC and middle temporal gyrus and left anterior cingulate gyrus and a CSF excess in areas close to the superior frontal gyrus bilaterally, right dorso-lateral PFC, left superior temporal gyrus and right precentral gyrus. Greater GM volume in the left dorsomedial PFC was associated with better working memory, attention and psychomotor speed in patients.

The relationships between longer duration of illness and lower GM volume in the left dorsomedial prefrontal and middle frontal cortices in patients with schizophrenia or schizoaffective disorder and CSF excess near the inferior frontal gyrus in patients with schizophrenia support the hypothesis that the frontal lobe may be vulnerable to the long-term effects of schizophrenia-related morbidity and findings from previous ROI studies [16,18]. The extended maturation period of the frontal lobe of up to 47 years [6] may create a larger window of vulnerability to abnormal neurodevelopment of selected areas of the frontal lobe among some schizophrenia patients. Our findings may simply reflect neurodegeneration with normal ageing, 
though normal ageing and duration of illness may be closely related and it may be difficult to separate the two effects. Age, in addition to duration of illness, was a significant predictor of GM volume in the dorsomedial PFC, suggesting that both factors may influence GM volume in the dorsomedial PFC in schizophrenia patients. The association between GM volume in the right middle frontal cortex and duration of illness was not influenced by age and GM volume in this region was not associated with age in controls, suggesting that this part of the frontal lobe may show more illness-specific effects than the PFC.

There was no direct association between duration of illness and neuropsychological function or symptom severity. However, greater GM volume in the left dorsomedial PFC was associated with better neuropsychological performance on measures that reflect frontal lobe functioning in patients. This would suggest that duration of illness effects are seen at the neural level, or are less sensitive to impairment at the neuropsychological and clinical levels of analysis. Duration of illness may not directly influence neuropsychological function, but the associated GM deficit in the left dorsomedial PFC may indirectly have a negative effect on frontal lobe-based neuropsychological function and is consistent with the evidence for the presence of such structure-function relationships in chronic patients [38-40]. A longer duration of illness may have a deleterious effect on frontal lobe-based structure-function relationships.

The association between longer duration of illness and lower GM volume and CSF excess in the cerebellum is consistent with an earlier report of an association between longer duration of illness and cerebellar GM volume using VBM in chronic patients [25], though it was not observed using the ROI approach in our previous study [16]. It is possible that the duration of illness effect may be localised to specific regions within the cerebellum. Deficits may be localized to certain areas that are related to cognitive function [41-43] and functional outcome [44]. Cerebellum has been traditionally considered in terms of its role in balance and motor coordination. Recent functional imaging, cerebellar lesion, animal studies and experimental 
studies in people with schizophrenia have widened its field of reference to other cognitive functions, in particular procedural learning and working memory [45-48]. The long-term effects of schizophrenia on the cerebellum may in part contribute to the observed cognitive deficits in the patients. Age was associated with a CSF excess near this region in controls, suggesting that normal ageing may also be associated with changes in this region.

We also observed an association between longer duration of illness and lower GM volume in the left $F G$ in patients and between age and lower $G M$ volume in the left $F G$ in controls. Age-related decline in brain activation in the FG during cognitive performance has been observed [49,50], suggesting that age-related decline may take place in the FG.

There was no overlap between localised areas showing an association between duration of illness and GM volume and those areas showing reduced GM volume or a CSF excess relative to healthy controls. This may be because significant group differences would be evident only in those regions that show lower GM volume in the majority of the patient sample.

Our study has some limitations. This was a retrospective study. The duration of illness construct is a proxy for the long-term effects of illness chronicity and may subsume more than just the long-term experience of schizophrenia. It may reflect longer contact with clinical services. Furthermore, the analyses of the association between duration of illness and neuropsychological function, clinical symptoms and GM volume were correlational in nature and thus do not inform about the direction of causality. We did not have reliable information to be able to examine the potential influence on GM volume of the duration of the period prodromal to the first frank illness episode, nor for the total length of medication in the observed brainduration of illness relationships; the age of onset of psychotic symptoms and age at first hospitalization/onset of antipsychotic medication, as reported by the patients, were very close in time in our sample. Finally, we did not control for alcohol, tobacco and illegal drug consumption that are known to influence cortical volumes [51,52]. 
In conclusion, the right middle frontal cortex may be vulnerable to the long-term effect of schizophrenia illness whereas both a long duration of illness and advance age influence the dorsomedial PFC, FG and cerebellum. The effect of illness chronicity on GM volume in the left dorsomedial PFC may be extended to frontal lobe brain structure-neuropsychological function relationships. The effect of illness chronicity on GM volume in the left dorsomedial PFC may be extended to brain structure-neuropsychological function relationships. 


\section{Acknowledgements}

The study was supported by funds from the Wellcome Trust, UK (067427/z/02/z). VK and PP are supported by a Wellcome Trust Senior Research Fellowship to Veena Kumari (067427/z/02/z). 


\section{References}

[1] Goldman-Rakic PS. The prefrontal landscape: implications of functional architecture for understanding human mentation and the central executive. Philosophical Transactions of the Royal Society of London - Series B: Biological Sciences, 1996; 351: 1445-1453.

[2] Northcutt RG, Kaas JH. The emergence and evolution of mammalian neocortex. Trends in Neurosciences, 1995; 18: 373-379.

[3] Shenton ME, Dickey CC, Frumin M, et al. A review of MRI findings in schizophrenia. Schizophrenia Research, 2001; 49: 1-52.

[4] Callicott JH, Bertolino A, Mattay VS, et al. Physiological dysfunction of the dorsolateral prefrontal cortex in schizophrenia revisited. Cerebral Cortex, 2000; 10: 1078-1092.

[5] Goldman-Rakic PS, Selemon LD. Functional and anatomical aspects of prefrontal pathology in schizophrenia. Schizophrenia Bulletin, 1997; 23: 437-458.

[6] Bartzokis G. Schizophrenia: breakdown in the well-regulated lifelong process of brain development and maturation. Neuropsychopharmacology, 2002; 27: 672-683.

[7] Ettinger U, Chitnis XA, Kumari $V$, et al. Magnetic resonance imaging of the thalamus in firstepisode psychosis. American Journal of Psychiatry, 2002; 158: 116-118.

[8] Steen RG, Mull C, McClure R, et al. Brain volume in first-episode schizophrenia: systematic review and meta-analysis of magnetic resonance imaging studies. British Journal of Psychiatry, 2006; 188: 510-518.

[9] Fannon D, Chitnis X, Dokum V, et al. Features of structural brain abnormality detected in first-episode psychosis. American Journal of Psychiatry, 2000; 157: 1829-1834.

[10] Fannon D, Tennakoon L, Sumich A, et al. Third ventricle enlargement and developmental delay in first-episode psychosis: preliminary findings. British Journal of Psychiatry, 2000b; 177: 354-359.

[11] Sumich A, Chitnis XA, Fannon DG, et al. Temporal lobe abnormalities in first-episode psychosis. American Journal of Psychiatry, 2002; 159: 1232-1234.

[12] Selemon LD, Kleinman JE, Herman MM, et al. Smaller frontal gray matter volume in postmortem schizophrenic brains. American Journal of Psychiatry, 2002; 159: 1983-1991.

[13] Bachmann S, Bottmer C, Pantel J, et al. MRI-morphometric changes in first-episode schizophrenic patients at 14 months follow-up. Schizophrenia Research, 2004; 67: 301303.

[14] Gur RE, Cowell P, Turetsky BI, et al. A follow-up magnetic resonance imaging study of schizophrenia. Relationship of neuroanatomical changes to clinical and neurobehavioral measures. Archives of General Psychiatry, 1998; 55: 145-152.

[15] Mathalon DH, Sullivan EV, Lim KO, et al. Progressive brain volume changes and the clinical course of schizophrenia in men: a longitudinal magnetic resonance imaging study. Archives of General Psychiatry, 2001; 58: 148-157.

[16] Premkumar P, Kumari V, Corr PJJ, et al. Frontal lobe volumes in schizophrenia: effects of stage and duration of illness. Journal of Psychiatric Research, 2006; 40: 627-637.

[17] Sapara A, Cooke M, Fannon D, et al. Prefrontal cortex and insight in schizophrenia: a volumetric MRI study. Schizophrenia Research, 2007; 89: 22-34. 
[18] Molina V, Sanz J, Sarramea F, et al. Lower prefrontal gray matter volume in schizophrenia in chronic but not in first episode schizophrenia patients. Psychiatry Research, 2004; 131 : 45-56.

[19] Molina V, Sanz J, Sarramea F, et al. Association between excessive frontal cerebrospinal fluid and illness duration in males but not in females with schizophrenia. European Psychiatry, 2005; 20: 332-338.

[20] Lopez-Garcia P, Aizenstein HJ, Snitz BE, et al. Automated ROI-based brain parcellation analysis of frontal and temporal brain volumes in schizophrenia. Psychiatry Research, 2006; 147: 153-161.

[21] Hietala J, Cannon TD, van Erp TG, et al. Regional brain morphology and duration of illness in never-medicated first-episode patients with schizophrenia. Schizophrenia Research, 2003; 64: 79-81.

[22] Molina V, Sanchez J, Reig S, et al. N-acetyl-aspartate levels in the dorsolateral prefrontal cortex in the early years of schizophrenia are inversely related to disease duration. Schizophrenia Research, 2005; 73: 209-219.

[23] van Haren NE, Hulshoff Pol HE, Schnack HG, et al. Focal gray matter changes in schizophrenia across the course of the illness: a 5-year follow-up study. Neuropsychopharmacology, 2007; 32: 2057-2066.

[24] Ananth H, Popescu I, Critchley HD, et al. Cortical and subcortical gray matter abnormalities in schizophrenia determined through structural magnetic resonance imaging with optimized volumetric voxel-based morphometry. American Journal of Psychiatry, 2002; 159: 14971505.

[25] Velakoulis D, Wood SJ, Smith DJ, et al. Increased duration of illness is associated with reduced volume in right medial temporal/anterior cingulate grey matter in patients with chronic schizophrenia. Schizophrenia Research, 2002; 57: 43-49.

[26] Bartzokis G, Beckson M, Lu PH, et al. Age-related changes in frontal and temporal lobe volumes in men: a magnetic resonance imaging study. Archives of General Psychiatry, 2001; 58: 461-465.

[27] Sullivan EV, Marsh L, Mathalon DH, et al. Age-related decline in MRI volumes of temporal lobe gray matter but not hippocampus. Neurobiology of Aging, 1995; 16: 591-606.

[28] Abe O, Yamasue H, Aoki S, et al. Aging in the CNS: comparison of gray/white matter volume and diffusion tensor data. Neurobiology of Aging, 2008; 29: 102-116.

[29] Alexander GE, Chen K, Merkley TL, et al. Regional network of magnetic resonance imaging gray matter volume in healthy aging. Neuroreport, 2006; 17: 951-956.

[30] Chen EY, Lam LC, Chen RY, et al. Prefrontal neuropsychological impairment and illness duration in schizophrenia: a study of 204 patients in Hong Kong. Acta Psychiatrica Scandinavica, 1996; 93: 144-150.

[31] Scully PJ, Coakley G, Kinsella A, et al. Psychopathology, executive (frontal) and general cognitive impairment in relation to duration of initially untreated versus subsequently treated psychosis in chronic schizophrenia. Psychological Medicine, 1997; 27: 1303-1310.

[32] Cuesta MJ, Peralta V, Zarzuela A. Illness duration and neuropsychological impairments in schizophrenia. Schizophrenia Research, 1998; 33: 141-150. 
[33] First MB, Spitzer RL, Gibbon M, et al. Structured Clinical Interview for DSM-IV-TR Axis I Disorders, Research Version, Non-patient Edition. (SCID-I/NP). New York, NY: Biometrics Research Department, 2002.

[34] First MB, Spitzer RL, Gibbon M, et al. Structured Clinical Interview for DSM-IV-TR Axis I Disorders - Patient Edition (SCID-I/P, 11/2002 revision). New York, NY: Biometrics Research Department, 2002.

[35] Kay SR, Fiszbein A, Opier LA. The Positive and Negative Syndrome Scale (PANSS) for schizophrenia. Schizophrenia Bulletin, 1987; 13: 261-276.

[36] Ashburner J, Friston KJ. Nonlinear spatial normalization using basis functions. Human Brain Mapping, 1999; 7: 254-266.

[37] Ashburner J, Friston KJ. Voxel-based morphometry--the methods. Neuroimage, 2000; 11: 805-21.

[38] Seidman LJ, Yurgelun-Todd D, Kremen WS, et al. Relationship of prefrontal and temporal lobe MRI measures to neuropsychological performance in chronic schizophrenia. Biological Psychiatry, 1994; 35: 235-246.

[39] Premkumar P, Kumari V, Corr PJ, et al. Neuropsychological function-brain structure relationships and stage of illness: An investigation into chronic and first-episode schizophrenia. Psychiatry Research, 2008; 162: 195-204.

[40] Baare WF, Hulshoff Pol HE, Hijman R, et al. Volumetric analysis of frontal lobe regions in schizophrenia: relation to cognitive function and symptomatology. Biological Psychiatry, 1999; 45: 1597-1605.

[41] Antonova E, Sharma T, Morris R, et al. The relationship between brain structure and neurocognition in schizophrenia: a selective review. Schizophrenia Research, 2004; 70 : 117-45.

[42] Andreasen NC, Pierson R. The Role of the Cerebellum in Schizophrenia. Biological Psychiatry, 2008.

[43] Picard H, Amado I, Mouchet-Mages S, et al. The role of the cerebellum in schizophrenia: an update of clinical, cognitive, and functional evidences. Schizophrenia Bulletin, 2008; 34 : 155-172.

[44] Wassink TH, Andreasen NC, Nopoulos P, et al. Cerebellar morphology as a predictor of symptom and psychosocial outcome in schizophrenia. Biological Psychiatry, 1999; 45: 4148.

[45] Chein JM, Fiez JA. Dissociation of verbal working memory system components using a delayed serial recall task. Cerebral Cortex, 2001; 11: 1003-1014.

[46] Chen SH, Desmond JE. Temporal dynamics of cerebro-cerebellar network recruitment during a cognitive task. Neuropsychologia, 2005; 43: 1227-1237.

[47] Desmond JE, Chen SH, Shieh PB. Cerebellar transcranial magnetic stimulation impairs verbal working memory. Annals of Neurology, 2005; 58: 553-560.

[48] Walter H, Vasic N, Hose A, et al. Working memory dysfunction in schizophrenia compared to healthy controls and patients with depression: evidence from event-related fMRI. Neuroimage, 2007; 35: 1551-1561.

[49] Kukolja J, Thiel CM, Wilms M, et al. Ageing-related changes of neural activity associated with spatial contextual memory. Neurobiology of Aging, 2007. 
[50] Madden DJ, Whiting WL, Provenzale JM, et al. Age-related changes in neural activity during visual target detection measured by fMRI. Cerebral Cortex, 2004; 14: 143-155.

[51] Pfefferbaum A, Sullivan EV, Mathalon DH, et al. Frontal lobe volume loss observed with magnetic resonance imaging in older chronic alcoholics. Alcoholism, Clinical and Experimental Research, 1997; 21: 521-529.

[52] Sim ME, Lyoo IK, Streeter CC, et al. Cerebellar gray matter volume correlates with duration of cocaine use in cocaine-dependent subjects. Neuropsychopharmacology, 2007; 32: 2229-2237.

[53] Wechesler D. Wechsler Abbreviated Scale of Intelligence (WASI) manual. San Antonio, Tx: Psychological Corporation, 1999.

[54] Heaton RK, Chelune GJ, Tally JL, et al. Wisconsin Card Sorting Test manual: Revised and Expanded. Odessa, FL: Psychological Assessment Resources, 1993.

[55] Burgess PW, Shallice T. The Hayling and Brixton Tests. Bury St Edmunds: Thames Valley Test Company, 1997.

[56] Milner B. Psychological aspects of focal epilepsy and it's neuropsychological management. In: Purpura DP, Penry JK and Walter RD, editors. Advances in Neurology. Vol 8. New York: Raven Press, 1975: 229-321.

[57] Gold JM, Carpenter C, Randolph C, et al. Auditory working memory and Wisconsin Card Sorting Test performance in schizophrenia. Archives of General Psychiatry., 1997; 54: 159-165.

[58] Wechsler D. Wechsler Memory Scale - Revised. New York: Psychological Corporation, 1987.

[59] Shapiro AM, Benedict RH, Schretlen D, et al. Construct and concurrent validity of the Hopkins Verbal Learning Test-revised. The Clinical Neuropsychologist, 1999; 13: 348-358.

[60] Cornblatt BA, Risch JJ, Faris G, et al. The Continuous Performance Test, Identical Pairs Version (CPT-IP): I. New findings about sustained attention in normal families. Psychiatry Research, 1988; 26: 223-238.

[61] Stroop JR. Studies of interference in serial verbal reactions. Journal of Experimental Psychology, 1935; 18: 643-662.

[62] Matthews CG, Klove H. Instruction Manual for the Adult Neuropsychology Test Battery. University of Wisconsin Medical School, WI, 1964. 
Table 1: Table of neuropsychological measures

\begin{tabular}{l} 
General intelligence \\
Wechsler Abbreviated Scale of Intelligence (WASI) Vocabulary and Matrix Design [53] \\
\hline Executive function \\
Wisconsin Card Sorting Test [54] \\
Hayling Test [55] \\
Verbal fluency [56] \\
\hline Working Memory \\
Letter-Number Test [57] \\
\hline Short-term/long-term memory \\
WMS-R Logical Memory [58] \\
Hopkins Verbal Learning Test (HVLT) [59] \\
\hline Attention \\
Continuous Performance Test - Identical Pairs [60] \\
Stroop Test [61] \\
\hline Psychomotor speed \\
Grooved Pegboard Test [62]
\end{tabular}


Table 2: Demographic and clinical characteristics of patients and healthy controls

\begin{tabular}{|c|c|c|c|c|}
\hline Characteristic & $\begin{array}{l}\text { Patient } \\
(\mathrm{n}=64)\end{array}$ & $\begin{array}{l}\text { Control } \\
(n=25)\end{array}$ & ANOVA $/ \chi^{2}$ & $p$ \\
\hline Age - mean, s.d., range & $38.6,9.6,19-61$ & $36.4,11.1,25-65$ & ${ }^{\dagger} 0.83$ & 0.37 \\
\hline Sex (male/female) & $48 / 16$ & $15 / 10$ & $\ddagger_{1.96}$ & 0.16 \\
\hline $\begin{array}{l}\text { Handedness (right-handed/ } \\
\text { ambidexterous) }\end{array}$ & 62,2 & 31,2 & $\ddagger_{2.29}$ & 0.13 \\
\hline $\begin{array}{l}\text { Years in education - mean, s.d., } \\
\text { range }\end{array}$ & $13.8,2.6,8-20$ & $15.2,2.6,10-20$ & ${ }^{\dagger} 4.63$ & 0.03 \\
\hline Diagnosis & & & & \\
\hline Schizophrenia & & & & \\
\hline Paranoid & 48 & & & \\
\hline Residual & 4 & & & \\
\hline Undifferentiated & 4 & & & \\
\hline Schizoaffective & 8 & & & \\
\hline Duration of illness - mean, s.d. & $13.7,9.7,1-43$ & & & \\
\hline $\begin{array}{l}\text { Age at illness onset - mean, s.d., } \\
\text { range }\end{array}$ & $24.9,8.0,10-50$ & & & \\
\hline $\begin{array}{l}\text { Symptoms (PANSS) - mean, s.d., } \\
\text { range }\end{array}$ & & & & \\
\hline Positive & $16.1,4.8,7-25$ & & & \\
\hline Negative & $17.7,5.0,7-28$ & & & \\
\hline Generalised psychopathology & $32.3,6.7,18-56$ & & & \\
\hline Total & $66.2,13.9,37-108$ & & & \\
\hline
\end{tabular}


Table 3: Comparison of neuropsychological performance between patients and controls controlling for age and years in education.

Neuropsychological measure

General intelligence

WASI IQ

Executive function

WCST perseverative errors

Hayling B time

Verbal fluency - letters

Verbal fluency - categories

Working memory

Letter-number

Short-term memory

HVLT total recalled

Logical memory immediate recall

Long-term memory

Logical memory delayed recall

Sustained Attention

Continuous Performance Test discriminability

Stroop Interference

Motor dexterity

Grooved pegboard - dominant

Grooved pegboard - non-dominant

\section{Patient}

n mean (s.d.) $\mathrm{n} \quad$ mean (s.d.)

\section{ANOVA}

$\mathrm{F}$
Effect

size

(partial

eta $\left.{ }^{2}\right)$

$63 \quad 103.3(20.1) \quad 25 \quad 119.6(15.2) \quad 13.46 \quad<0.001 \quad 0.14$

$\begin{array}{lllllll}64 & 24.5(17.6) & 22 & 11.9(9.5) & 10.17 & 0.002 & 0.11\end{array}$

$\begin{array}{lllllll}61 & 36.9(36.2) & 24 & 26.9(39.8) & 1.25 & 0.3 & 0.02\end{array}$

$\begin{array}{lllllll}64 & 36.1(13.1) & 23 & 48(17.5) & 11.52 & 0.001 & 0.12\end{array}$

$\begin{array}{lllllll}64 & 39.4(11.2) & 23 & 53(15.1) & 20.56 & <0.001 & 0.2\end{array}$

$\begin{array}{lllllll}64 & 13.0(4.2) & 22 & 9.9(3.2) & 11.55 & 0.001 & 0.12\end{array}$

$\begin{array}{lllllll}63 & 20.6(5.8) & 22 & 27.3(4.5) & 24.7 & <0.001 & 0.23\end{array}$

$\begin{array}{lllllll}63 & 6.6(3.1) & 22 & 9.8(3.2) & 18.33 & <0.001 & 0.18\end{array}$

$\begin{array}{lllllll}63 & 7.6(2.5) \quad 22 & 10.5(3.2) & 18.33 & <0.001 & 0.18\end{array}$

$\begin{array}{lllllll}62 & 0.9(0.6) & 21 & 1.5(0.8) & 15.44 & <0.001 & 0.16\end{array}$

$\begin{array}{lllllll}62 & 0.02(9.3) & 21 & 3.1(15.3) & 1.2 & 0.3 & 0.02\end{array}$

$\begin{array}{lllllll}63 & 94.4(63.7) & 24 & 62.4(14) & 5.9 & 0.02 & 0.07\end{array}$

$\begin{array}{lllllll}63 & 113.4(86.3) & 24 & 76.3(20) & 4.3 & 0.04 & 0.05\end{array}$ 
Table 4. Brain areas showing GM reduction and CSF excess in patients relative to healthy controls correcting for age (height threshold $p=0.001$, cluster size $>25$ voxels)

\begin{tabular}{|c|c|c|c|c|c|c|c|c|c|}
\hline \multirow[t]{2}{*}{ Brain Region } & \multirow{2}{*}{$\begin{array}{l}\text { Left/ } \\
\text { right }\end{array}$} & \multirow{2}{*}{$\begin{array}{c}\text { Brodmann } \\
\text { Area L/R }\end{array}$} & \multicolumn{3}{|c|}{$\begin{array}{c}\text { MNI } \\
\text { Coordinates }\end{array}$} & \multirow{2}{*}{$\begin{array}{c}t \\
\text { value }\end{array}$} & \multirow{2}{*}{$\begin{array}{l}\text { Number of } \\
\text { contiguous } \\
\text { voxels }\end{array}$} & \multirow{2}{*}{$\begin{array}{l}\text { Family- } \\
\text { wise error } \\
\text { corrected } \\
\text { p-value }\end{array}$} & \multirow{2}{*}{$\begin{array}{l}\text { Voxel } \\
\text { equiv } \\
Z\end{array}$} \\
\hline & & & $\mathbf{x}$ & $y$ & $\mathbf{z}$ & & & & \\
\hline GM reduction & & & & & & & & & \\
\hline Dorsolateral PFC & $\mathrm{R}$ & 46 & 38 & 41 & 27 & 4.99 & 2715 & $<0.001$ & 4.66 \\
\hline Dorsolateral PFC & L & 46 & -33 & 41 & 19 & 3.75 & 390 & 0.004 & 3.6 \\
\hline Anterior cingulate gyrus & L & 24 & 1 & -12 & 39 & 4.09 & 2613 & 0.002 & 3.9 \\
\hline Middle temporal gyrus & L & 21 & -52 & -41 & 2 & 4.03 & 943 & 0.002 & 3.84 \\
\hline Middle temporal gyrus & $\mathrm{R}$ & 1 & 48 & -47 & 24 & 4.37 & 755 & 0.001 & 4.14 \\
\hline CSF excess adjacent to & & & & & & & & & \\
\hline Dorsolateral PFC & $\mathrm{R}$ & & 51 & 34 & -17 & 5.29 & 2282 & $<0.001$ & 4.91 \\
\hline Superior frontal gyrus & $\mathrm{R}$ & & 22 & 28 & 55 & 4.27 & 509 & 0.001 & 4.05 \\
\hline Superior temporal gyrus & L & & -57 & 18 & -25 & 3.88 & 512 & 0.004 & 3.71 \\
\hline Superior frontal gyrus & $\mathrm{R}$ & & 6 & 12 & 58 & 4.01 & 1056 & 0.003 & 3.83 \\
\hline Precentral gyrus & $\mathrm{R}$ & & 24 & 8 & 83 & 3.38 & 80 & 0.019 & 3.26 \\
\hline Superior frontal gyrus & L & & -7 & -1 & 66 & 3.52 & 34 & 0.012 & 3.39 \\
\hline
\end{tabular}

PFC: Prefrontal cortex; MNI: Montreal Neurological Institute. 
Table 5: Brain areas showing an association between duration of illness and GM volume and CSF in patients with schizophrenia or schizoaffective disorder (height threshold $p<0.001$, cluster size $>25$ voxels)

\begin{tabular}{|c|c|c|c|c|c|c|c|c|}
\hline \multirow{2}{*}{ Brain Region } & \multirow{2}{*}{$\begin{array}{l}\text { Left/ } \\
\text { right }\end{array}$} & \multirow{2}{*}{$\begin{array}{c}\text { Brodmann } \\
\text { Area L/R }\end{array}$} & \multicolumn{2}{|c|}{$\begin{array}{c}\text { MNI } \\
\text { Coordinates }\end{array}$} & \multirow{2}{*}{$\begin{array}{c}t \\
\text { value }\end{array}$} & \multirow{2}{*}{$\begin{array}{l}\text { Number of } \\
\text { contiguous } \\
\text { voxels }\end{array}$} & \multirow{2}{*}{$\begin{array}{c}\text { Family- } \\
\text { wise error } \\
\text { corrected } \\
\text { p-value }\end{array}$} & \multirow{2}{*}{$\begin{array}{c}\text { Equiv } \\
Z\end{array}$} \\
\hline & & & & $\mathbf{z}$ & & & & \\
\hline
\end{tabular}

Inverse association between duration of illness and GM

$\begin{array}{lllrrrrrrr}\text { Dorsomedial PFC } & \mathrm{L} & 8 & -2 & 49 & 45 & 3.89 & 186 & 0.004 & 3.66 \\ \text { Middle frontal cortex } & \mathrm{R} & 6 / 8 & 35 & 7 & 49 & 3.52 & 96 & 0.01 & 3.35 \\ \text { Fusiform gyrus } & \mathrm{L} & 37 & -41 & -37 & -18 & 3.53 & 98 & 0.01 & 3.36 \\ \text { Cerebellum (lobule III) } & \mathrm{L} & & -52 & -46 & -30 & 3.43 & 134 & 0.013 & 3.27\end{array}$

Positive association between duration of illness and CSF excess adjacent to

Cerebellum

$\begin{array}{llll}-11 & -28 & -15 & 4.20\end{array}$

492

0.002

3.92

Inverse association between duration of illness and GM in patients with a schizophrenia diagnosis only $(n=56)$

$\begin{array}{lrrrrrrrrr}\text { Dorsomedial PFC } & \mathrm{L} & 8 & -17 & 46 & 41 & 4.36 & 1178 & 0.001 & 4.02 \\ \text { Middle frontal cortex } & \mathrm{R} & 6 & 35 & 7 & 49 & 4.05 & 387 & 0.003 & 3.77\end{array}$

Positive association between duration of illness and CSF excess in patients with a schizophrenia diagnosis only ( $\mathrm{n}$ $=56$ )

Inferior frontal gyrus

L $\quad 28$

$\begin{array}{llll}-19 & 7 & -21 & 3.99\end{array}$

327

0.005

3.72 
Figure 1: Areas showing reduced GM in patients relative to healthy controls (height threshold $p=0.001$ )

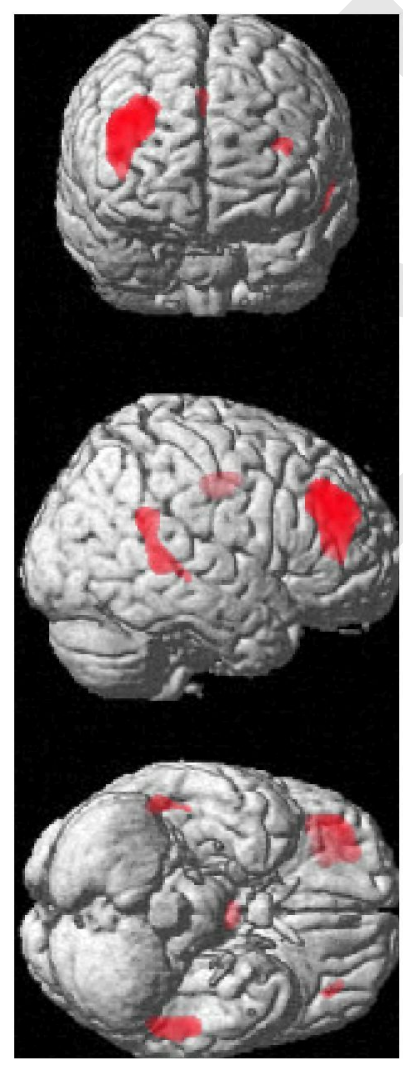


Figure 2: Inverse associations between duration of illness and GM volume in patients (height threshold $p=0.002$ )

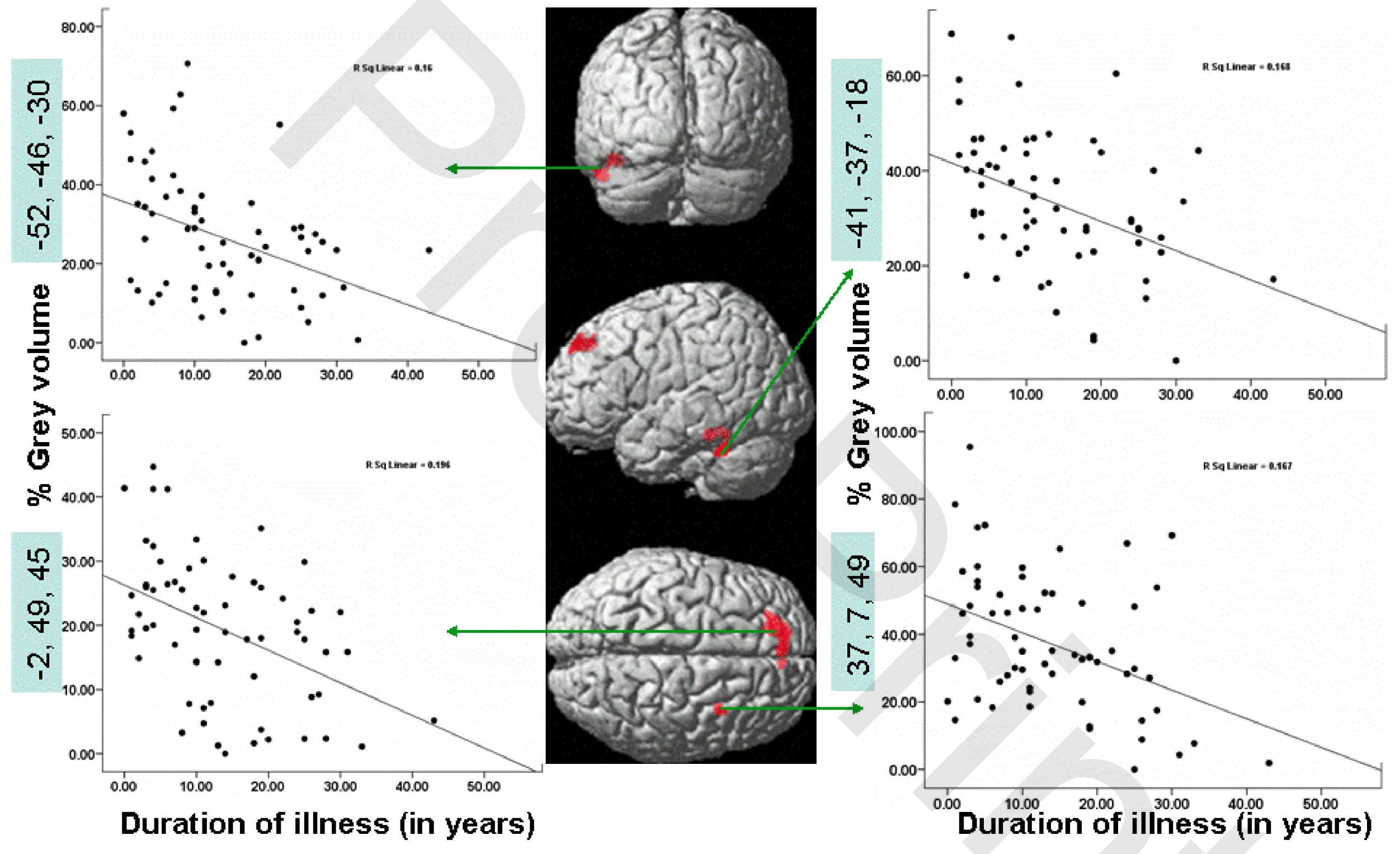

